\title{
Evolution of mineralogy and porosity of cement-clay interfaces
}

\author{
P. LURASCHI ${ }^{1,2^{*}}$, T.GIMMI ${ }^{1,2}$
}

${ }^{1}$ Laboratory for Waste Management, PSI, CH-5232 Villigen

PSI, Switzerland (*correspondence:

pietro.luraschi@psi.ch)

${ }^{2}$ Institute of Geological Sciences, University of Bern, CH-

3012 Bern, Switzerland (gimmi@geo.unibe.ch)

According to the Swiss multiple barrier concept for a deep geological repository for radioactive waste, cement and clay will come in contact at several places (e.g., cement tunnel liner and Opalinus Clay host rock or bentonite clay barrier, or mortar used as cavern back fill and Opalinus Clay). The strong difference in chemical composition of cement and clay materials is reflected in the respective porewaters. Accordingly, a strong chemical disequilibrium exists at an interface between cement and clay that leads to dissolution/precipitation reactions, which may then affect safety relevant properties of the repository (e.g., gas transport, solute transport, resaturation). The typically small extension of alterations ( $\mathrm{mm}$ to $\mathrm{cm}$ scale) and the mechanical fragility of such interfaces makes their experimental investigation challenging. The development of a special cell allowed studying the alterations in small cement-clay samples over time. To simplify the system, a high porosity hardened cement paste and Na-montmorillonite were used as interface components [1].

Once created the cement-clay interfaces were saturated with the corresponding porewater solutions and let interact for several years under nitrogen atmosphere (to reduce carbonation). During this period the samples were repeatedly investigated by means of neutron imaging at SINQ (PSI). This technique allowed monitoring non-destructively the evolution of the water content at the interface (which in a saturated medium can be used as a proxy for porosity). Subsequently the samples were destructively investigated by means of different techniques (SEM/EDX, XRD, and TGA) to characterize the chemical and the mineralogical modifications that occurred at the interfaces.

[1] Shafizadeh et al., 2020. Cement and Conrete research, $127,105924$. 